\title{
Foscolo, Manzoni e la cerchia di Byron: la prima ricezione inglese della Ricciarda e del Carmagnola
}

Foscolo, Manzoni et l'entourage de Byron : la première réception anglaise de Ricciarda e de Carmagnola

Foscolo, Manzoni and Byron's Circle: The Early English Reception of Ricciarda and Carmagnola

\section{Paolo Borsa e Christian Del Vento}

\section{(2) OpenEdition}

\section{Journals}

Edizione digitale

URL: http://journals.openedition.org/cei/2423

DOI: $10.4000 /$ cei.2423

ISSN: 2260-779X

\section{Editore}

UGA Éditions/Université Grenoble Alpes

\section{Edizione cartacea}

Data di pubblicazione: 30 juin 2015

Paginazione: 139-154

ISBN: 978-2-84310-289-9

ISSN: 1770-9571

Notizia bibliografica digitale

Paolo Borsa e Christian Del Vento, «Foscolo, Manzoni e la cerchia di Byron: la prima ricezione inglese della Ricciarda e del Carmagnola», Cahiers d'études italiennes [Online], 20 | 2015, online dal 01 janvier 2017, consultato il 26 mars 2021. URL: http://journals.openedition.org/cei/2423 ; DOI: https://doi.org/ $10.4000 /$ cei.2423 


\title{
FOSCOLO, MANZONI E LA CERCHIA DI BYRON:
}

\section{LA PRIMA RICEZIONE INGLESE DELLA RICCIARDA E DEL CARMAGNOLA}

\author{
Paolo Borsa, Christian Del Vento* \\ Università di Milano, Université Sorbonne Nouvelle Paris 3
}

In un diario inedito portato alla luce da Patrick Labarthe, Charles-Augustin de Sainte-Beuve annotava, in data II dicembre I847, una conversazione avuta con Victor Cousin, nella quale il filosofo dava conto dell'entusiasmo suscitato in Goethe dalla lettura del Conte di Carmagnolar. Secondo Cousin, Goethe non solo avrebbe annunciato Manzoni all'Europa, ma lo avrebbe in seguito difeso dalle aspre critiche, non altrimenti note, a lui mosse da Foscolo su una non meglio precisata rivista inglese (il sintagma «une Revue anglaise» sostituisce il riferimento, cassato, all'autorevole «Edinburgh Review» di Francis Jeffrey) ${ }^{2}$ :

Pour Manzoni qu'il [Goethe] ne connaissait nullement, quand le Comte de Carmagnola lui tomba entre les mains, le voilà qui s'éprend, qui s'enfonce dans l'étude de cette pièce, qu'il y découvre mille intentions, mille beautés, et un jour dans son journal (sur l'art et l'antiquité) où il déversait ses pensées pour se soulager, il annonce Manzoni à l'Europe. Quand Foscolo dans la Reve anglaise l'attaqua, il le défendit et par toutes sortes de raisons auxquelles Manzoni n’avait certes pas songé.

Com'è noto, il Carmagnola, pubblicato nel gennaio I820 con dedica a Claude Fauriel, fu spedito a Goethe, fresco di stampa, per il tramite di

\footnotetext{
L'articolo si intende come il risultato di un lavoro di stretta collaborazione e di una perfetta intesa tra gli autori. Desideriamo ringraziare l'amico e collega Patrick Labarthe che lavora attualmente all'edizione del Cahier brun e che, sottoponendo nell'autunno del 2012 a Christian Del Vento un passaggio del diario di SainteBeuve per un parere su un'allusione a Foscolo e al suo ruolo nella polemica sorta intorno alla pubblicazione del Conte di Carmagnola, è all'origine di questo nostro contributo.

I. Si veda il ms. D 57I della Bibliothèque de l'Institut de France di Parigi, Cahier brun, p. 26, di cui Patrick Labarthe prepara attualmente l'edizione in collaborazione con Bénédicte Élie per l'editore Droz.

2. Che Victor Cousin avesse fatto riferimento alla "Quarterly Review» e non all' «Edinburgh Review», come indicato da Sainte-Beuve nel suo diario, testimonia un breve passaggio dei Fragments et souvenirs di Cousin, dove lo scrittore francese racconta le sue visite a Goethe; $\mathrm{cfr}$. V. Cousin, Fragments et souvenirs, troisième édition considérablement augmentée, Paris, Didier, 1857, p. 159.
} 
Gaetano Cattaneo ${ }^{3}$. La rivista cui fa riferimento il diario di Sainte-Beuve è "Über Kunst und Alterthum», sulla quale Goethe, tra il I820 e il I82I, in tre articoli recensì positivamente gli Inni sacri e appunto il Carmagnola ${ }^{4}$. La nota di Sainte-Beuve può essere messa parzialmente in relazione con un'altra notizia ricavabile dai Colloquii col Manzoni del Tommaseo, secondo il quale, in una conversazione del I855, Manzoni «gli avrebbe detto d'essere stato nei primi anni ammiratore del Foscolo, ma che questa ammirazione non era "durata troppo" e che di questo il Foscolo si vendicò, "scrivendo in giornali inglesi del male di lui"» in relazione alla sua produzione tragicas.

Alla luce dello specifico contesto letterario e culturale in cui si collocano la pubblicazione del Carmagnola di Manzoni e della Ricciarda di Foscolo (composta in Italia tra il I8I2 e il I8I3, ma data alle stampe in Inghilterra solo nel maggio $1820^{6}$, poco dopo la prima tragedia manzoniana), l'obiettivo di questo contributo è, in primo luogo, identificare quale sia l'articolo «inglese» sfavorevole al Manzoni, cui fa riferimento Cousin nella nota di Sainte-Beuve, quindi verificare se esso sia ascrivibile a Foscolo o ad altro autore e, in questo caso, se sia possibile ipotizzare una collaborazione di Foscolo alla redazione del testo. È evidente che l'articolo in questione non può essere lo scritto noto come Della nuova scuola drammatica italiana: non solo perché esso risale agli ultimi anni dell'esilio in Inghilterra di Foscolo, ma anche perché restò incompiuto e, dunque, mai tradotto e pubblicato su una rivista britannica. Tale scritto, in realtà anepigrafo e rimasto tra le carte del poeta poi confluite presso la Biblioteca Labronica di Livorno, semmai poté essere all'origine della confidenza di Manzoni a Tommaseo del I855, poiché esso era stato reso pubblico per la prima volta solo pochi anni prima, nel I850, nella serie delle Opere edite $e$ postume del poeta, a cura dell'Orlandini, che erroneamente lo diceva «stampato per la prima volta in inglese nel primo numero della "Foreign Quarterly Review"»7. Una lunga recensione all'edizione Molini (I825) delle tragedie manzoniane (che conteneva anche altri versi del poeta e, in appendice, la Lettre di Manzoni a Victor Chauvet e gli scritti critici di

3. G. Gaspari, Goethe traduttore di Manzoni, in Premio "città di Monselice» per la traduzione letteraria e scientifica 28-29-30, Padova, Il Poligrafo, 2003, pp. 233-244, in particolare p. 234.

4. Ivi, pp. 233-235.

5. G. Gambarin, Ancora del Foscolo e del Manzoni, "Giornale storico della letteratura italiana», CXXXIX, I962, $\mathrm{n}^{\circ} 425$, pp. 7I-83, in particolare p. 75 .

6. Ricciarda. Tragedia di Ugo Foscolo, Londra, per J. Murray, I820. Per cui si veda l'introduzione di G. Bèzzola a EN II, pp. XviII-L, e la Scheda introduttiva di M. M. Lombardi in U. Foscolo, Opere, edizione diretta da F. Gavazzeni, vol. I, Torino, Einaudi-Gallimard, I994, pp. 876-884.

7. Avvertenza, in U. Foscolo, Prose letterarie, vol. IV, Firenze, Le Monnier, I850, p. 262. 
Goethe, Fauriel e Visconti) ${ }^{8}$ apparve, in effetti, sulla «Foreign Quarterly Review» del I827, adespota e con l'intitolazione, apposta sull'intestazione delle pagine, Italian Tragedy ${ }^{9}$. Lo scritto, molto interessante anche se trascurato dal dibattito critico sul periodo storico-letterario in oggetto, non può essere messo in relazione con l'inedito saggio foscoliano, né ci pare che vi si possa riconoscere una parte del Foscolo, a dispetto dei giudizi poco lusinghieri espressi sul Manzoni tragico (come quello, posto in chiusura, di avere agito con «a prudence that seems to border on cowardice» ${ }^{10}$ ) e benché le opinioni sulle unità tragiche e sul rapporto tra storia e poesia siano in qualche modo consonanti con quelle del poeta. Nel parlare con Tommaseo tuttavia - e ciò ci sembra che giustificherebbe una rinnovata attenzione per l'articolo - è possibile che Manzoni facesse riferimento proprio a questo scritto inglese, che avrebbe attribuito a Foscolo sulla base di quanto affermato da Orlandini nella sua Avvertenza.

Come che sia, l'articolo inglese che accese l'animo di Goethe deve essere riconosciuto, invece, in un lungo contributo, già segnalato da Gambarin ${ }^{\text {II }}$, identificato dall'intestazione Italian Tragedy (che, forse non a caso, sarebbe stata ripresa dall'articolo del ' 27 uscito sulla «Foreign Quarterly Review», di cui s'è appena detto), stampato sul numero di ottobre (ma pubblicato nel dicembre) del 1820 della prestigiosa "Quarterly Review»" ${ }^{\text {I2 }}$ Si tratta della stessa rivista sulla quale, l'anno prima, era apparso il contributo foscoliano dedicato ai Narrative and Romantic Poems of the Italians e in cui sarebbe stato pubblicato, al principio dell'anno successivo, il primo saggio petrarchesco dello stesso Foscolo. La «Quarterly Review» faceva capo a John Murray di Albemarle Street, l'editore londinese di riferimento di Lord Byron. Per i suoi tipi, nel I8I8, era uscito in appendice alle Historical Illustrations of the Fourth Canto of Childe Harold dello stesso Byron l'Essay on the Present Literature of Italy, frutto della collaborazione tra John Cam Hobhouse e Foscolo; e, nel maggio del I820, lo scrittore aveva dato alle stampe la Ricciarda e progettava di pubblicare il volume Narrative of Events Illustrating the Fortunes and Cession of Parga, poi accantonato nell'ottobre di quello stesso anno per motivi di opportunità politica. Sempre per John Murray sarebbe uscita, nell'inverno del I823, la seconda

\footnotetext{
8. Tragedie di Alessandro Manzoni milanese il Conte di Carmagnola e l'Adelchi. Aggiuntevi le poesie varie dello stesso, ed alcune prose sulla teoria del dramma tragico, Firenze, presso Giuseppe Molini all'insegna di Dante, I825.

9. Italian Tragedy, «The Foreign Quarterly Review», I, July I827, pp. I35-I70.

Io. Ivi, p. I68.

II. G. Gambarin, Ancora del Foscolo, cit., pp. 75-76.

I2. Italian Tragedy, «The Quarterly Review», XXIV, October I820, pp. 72-IO2.
} 
edizione, venale, degli Essays on Petrarch ${ }^{\text {13 }}$. Com'era tradizione nelle riviste periodiche trimestrali inglesi, l'articolo di cui ci occupiamo fu pubblicato adespota e anepigrafo (anche se provvisto nell'intestazione delle pagine dell'intitolazione Italian Tragedy) e si presenta come una recensione di tre tragedie contemporanee, il Conte di Carmagnola di Manzoni, la Ricciarda di Foscolo e la Francesca da Rimini di Pellico. Si tratta di un'informata e intelligente rassegna retrospettiva sulla tragedia italiana, dalle origini fino all'epoca presente. Essa si basa, per la prima parte, sul Teatro italiano di Scipione Maffei e sui primi nove tomi del Teatro italiano antico, usciti a Milano per i tipi della Società tipografica de' Classici Italiani tra il I808 e il I809, oltre che sull'unico lavoro in lingua inglese sulla tragedia italiana che fosse utilmente consultabile a quei tempi, ossia il volume Historical memoir on Italian tragedy di Joseph Cooper Walker, considerato un'opera scrupolosa ma poco affidabile, a causa della sua profusione e del suo atteggiamento indiscriminatamente elogiativo della tradizione italiana ${ }^{\text {I4 }}$. In estrema sintesi, la tesi dell'autore è che gli italiani, nella scelta di continuare a proporre nelle tragedie i soggetti classici, abbiano trascurato il loro «National drama» e i soggetti, potenzialmente efficaci sul piano tragico, tratti dalla loro storia nazionale («the Italians should look at home for their tragic subjects. Why should not Dante be to them what Homer was to the Greek tragedians?») ${ }^{\text {Is}}$; la causa di ciò doveva essere cercata nelle peculiari circostanze storiche e nelle croniche divisioni d'Italia, da cui dipende, soprattutto nei secoli XVII e XVIII, la generale decadenza culturale della nazione. Il punto di vista dell'autore inglese sul teatro italiano contemporaneo è consono a una prospettiva che si potrebbe definire latamente 'byroniana', nel senso che egli si fa promotore di un modello teatrale a un tempo classicista, romantico e nazionale.

Di là dall'esperienza tragica di Alfieri e dell'Aristodemo di Monti, arrivando all'esame delle opere dei principali tragediografi italiani viventi l'estensore di Italian Tragedy assegna la palma dell'eccellenza tragica al Foscolo della Ricciarda e, forse con una leggera preferenza, al Pellico della Francesca da Rimini, a causa del rispetto delle unità aristoteliche - che vengono considerate in qualche modo necessarie a fornire una regola al

13. Essays on Petrarch by Ugo Foscolo, London, John Murray, I823. Per cui si veda l'introduzione di C. Foligno a EN X, pp. XxI-XLVII, e la Scheda introduttiva di G. Lavezzi in U. Foscolo, Opere, cit., vol. II, I995, pp. IO28-IO37.

I4. [J. Cooper Walker], Historical memoir on Italian tragedy, from the earliest period to the present time, illustrated with specimens and analyses of the most celebrated tragedies... and biographical notices of the principal tragic writers of Italy, by a member of the Arcadian Academy of Rome [Eubante Tirinzio], London, printed for E. Harding, I799.

I5. Ivi, pp. IOO-IOI. 
'disordinato' e passionale genio italico - e della scelta di soggetti storici o storicizzanti, ossia conformi alla tradizione 'shakespeariana'. Una severa stroncatura è riservata invece al Carmagnola, bollato come una "feeble tragedy» priva di poesia e non meritevole di considerazione, benché il coro alla fine del secondo atto, interamente tradotto in inglese, sia degno delle «splendid odes» del suo autore e sia nell'articolo definito «the most noble piece of Italian lyric poetry which the present day has produced». La stessa violazione delle unità aristoteliche, cui Manzoni 'dichiara guerra' nella prefazione alla tragedia, appare all'estensore ben poca cosa se raffrontata alla libertà drammatica propria della tradizione inglese, fondata sull'autorevole esempio di Shakespeare e sugli argomenti di Johnson; sicché egli giudica il Carmagnola un esperimento insufficiente a persuadere gli autori italiani ad abbandonare le regole classiche («we fear [...] that the Italians will require a more splendid violation of their old established laws, before they are led to abandon them») ${ }^{16}$.

Sulla paternità 'ufficiale' di questo articolo non sussistono dubbi: l'autore fu il reverendo Henry Hart Milman, uomo di cultura, poeta, storico, tragediografo (nel I8Is aveva pubblicato la tragedia Fazio, portata in scena per la prima volta, senza il suo consenso, al Surrey Theatre con il titolo The Italian Wife e poi, con il titolo corretto, al Covent Garden nel I8I8) e, a partire dal I82I, professore di poesia all'università di Oxford ${ }^{17}$. La notizia può evincersi, anzitutto, da un passaggio della lettera di Mary Graham a Foscolo del 28 gennaio I82I, nella quale la Graham indica la traduzione dei brani della Ricciarda pubblicati nella "Quarterly Review» come opera del Milman, cui sarebbe dunque da ascrivere anche la lusinghiera recensione della tragedia ${ }^{18}$. Che fosse Milman l'autore dell'articolo è testimoniato, ottant'anni più tardi, dal figlio Arthur, che nel profilo biografico del padre riconosce l'articolo Italian Tragedy come il primo scritto di una costante e più che quarantennale collaborazione con la «Quarterly Review» del Murray (per i cui tipi, tra l'altro, Arthur Milman pubblicò il

I6. Italian Tragedy, I820, cit., p. 87.

17. Sul Milman oltre alla voce dell'Oxford Dictionary of National Biography, si vedano almeno, sub voce: A. Cunningham, Biographical and critical history of the British literature of the last lifty lears, by..., Paris, Baudry, I834; C. Knight, The English cyclopaedia, a new dictionary of universal knowledge, conducted by..., Biography, vol. 3, London, Bradbury and Evans, 1856; S. Austin Allibone, A critical dictionary of English literature and British and American authors living and deceased from the earliest accounts to the latter half of the nineteenth century by..., London, Trubner; Philadelphia, J. B. Lippincott \& Co, I859-I87I, vol. II; E. Creasy, Memoirs of eminent Etonians, by... A new edition..., London, Chatt, 1876; E. F. Hatfield, The poets of the church. A series of biographical sketches of hymn-writers, with notes on their hymns, New York, A. D. F. Randolph \& Co., I884.

I8. Ep. VIII, pp. 237-239, p. 239 per la citazione. 
Biographical Sketch del padre) ${ }^{\text {I9. }}$. La notizia è indirettamente confermata da John Murray III, figlio del fondatore della casa editrice. Quattro anni prima dell'uscita del volume di Arthur Milman, nel luglio del I896, il Murray aveva riferito a Richard Garnett «keeper of printed books of the British Museum», a sua volta consultato da Joel Foote Bingham traduttore della Francesca da Rimini, che non vi era motivo di dubitare che la versione inglese degli squarci della tragedia del Pellico inseriti nell'articolo Italian Tragedy del I820 fossero da attribuire al Milman, autore dello scritto $^{20}$. La precisazione del Murray si inseriva in una piccola querelle intorno alla paternità della prima (parziale) traduzione della Francesca da Rimini, sorta a causa di un'affermazione di Pellico - contenuta in una lettera al padre del 2I gennaio I82I e accolta nel Disegno storico della letteratura italiana del Fornaciari (I874) e, poi, nel Manuale della letteratura italiana di D'Ancona e Bacci (I892) - secondo cui la traduzione dei passi della sua tragedia sarebbe stata eseguita da Lord Byron ${ }^{21}$. Sulla vicenda, intricata, della versione della Francesca ritorneremo più avanti sulla base degli studi di Beatrice Corrigan e Angeline Lograsso ${ }^{22}$. Per il momento, basti qui rilevare come l'eventualità di un traduttore diverso dal Milman, estensore dello scritto, apra uno spiraglio alla possibilità che Italian Tragedy fosse, almeno in parte, il frutto di una collaborazione a più mani. Questa circostanza, messa in relazione con le testimonianze di Cousin e di Manzoni, porterebbe a riprendere in considerazione anche il nome di Foscolo per la questione dell'ideazione e stesura dell'articolo. Ad appena due anni di distanza, l'iniziativa si sarebbe configurata allora come una sorta di replica, solo un po' più complessa, dell'operazione che tante polemiche aveva suscitato in Italia tra i romantici, primo fra tutti il Di Breme, realizzata per l'Essay on the Present Literature of Italy, pubblicato a nome di Hobhouse in appendice alle Historical Illustrations del canto IV

19. A. Milman, Henry Hart Milman D. D. Dean of St Paul's. A Biographical Sketch, London, John Murray, 1900, p. 75. La notazione di Arthur Milman va messa in relazione con quanto William Gifford, direttore della rivista, scriveva al Murray in una lettera erroneamente datata "June I82I», dove si fa riferimento alle bozze fresche di stampa dell'articolo; cfr. H. Shine and H. Chadwick Shine, The Quarterly Review under Gifford. Identification of Contributors, Chapel Hill, University of North Carolina Press, 1949, p. 71, e ora anche l'utile risorsa online Quarterly Review Archive, edited by J. Cutmore, XXIV, 47 (October 1820), ospitata su Romantic Circles: beta. A refereed scholarly Website devoted to the study of Romantic-period literature and culture, e consultabile all'indirizzo: <http://www.rc.umd.edu/reference/qr/index/47.html>.

20. S. Pellico, Francesca da Rimini. A Tragedy... Translated in English Verse with Critical Preface and Historical Introduction by the Rev. J. F. Bingham, Cambridge (Mass.), Charles W. Seaver, I897, p. xxxv; cfr. B. Corrigan, Pellico's "Francesca da Rimini»: The First English Translation, «Italica», XXXI, 1954, n 4, pp. 215-224, in particolare p. 215 .

2I. B. Corrigan, Pellico's «Francesca da Rimini», cit., p. 215. La lettera di Pellico si legge in S. Pellico, Epistolario, Milano, Francesco Pagnoni, I873, p. I4.

22. A. Lograsso, Byron traduttore del Pellico, "Lettere italiane», XI, I959, n 2, pp. 234-249. 
del Childe Harold di Byron ${ }^{23}$. La tentazione è forte, tanto più che, chiudendo l'articolo, il Milman si rivolge direttamente a Foscolo, ormai da tempo residente tra i suoi connazionali: "To Signor Foscolo, who is resident amongst us, we may address ourselves more personally». Lodato come squisito conoscitore della lingua e della cultura greche e latine e come eccellente autore in lingua italiana, Foscolo è invitato a lasciare alla posterità un'opera più grande dell'Ortis e della Ricciarda, mentre la sua figura di esule è legata alle disilluse speranze di una riscossa nazionale italiana, al momento inimmaginabile, ma forse possibile in futuro, quando letterati e poeti saranno in grado di mettere il proprio genio al servizio dell'innalzamento del livello culturale e morale del popolo italiano:

[...] should a more fortunate period of her history unexpectedly arrive, if her poets, and men of letters shall have consecrated their powers to her improvement and instruction; if they have not only adorned her by their fame, but enlightened her by their generous principle; if they have not only raised her standard of intellectual, but also of moral greatness ${ }^{24}$.

La conoscenza diretta tra i due uomini di lettere è testimoniata, un anno e mezzo dopo la pubblicazione dell'articolo sulla "Quarterly Review», dalle lettere di presentazione che Foscolo consegnò a Milman in occasione di un suo viaggio in Italia nell'estate del $1822^{25}$. Milman, inoltre, avrebbe collaborato con Foscolo alla seconda edizione degli Essays on Petrarch, pubblicata tra febbraio e marzo del I823, per la quale s'incaricò di alcune delle traduzioni inglesi poste in appendice al volume ${ }^{26}$. Il testo

23. Sulla quale si vedano, oltre al classico studio di C. Calcaterra, La polemica Hobhouse-Di Breme e l'"Essay on the Present Literature of Italy» del I8I8, "Convivium», XVIII, I950, n ${ }^{\circ} 3$, pp. 32I-332; l'introduzione di C. Foligno a EN XI/I, pp. LxxIX-LxxxIx; la nota introduttiva di F. Gavazzeni in U. Foscolo, Opere, MilanoNapoli, Ricciardi, vol. II, I98I, pp. I397-I402; N. Havely, "This Infernal Essay»: English Context for Foscolo's "Essay on the Present Literature of Italy", in Immaginando l'Italia: itinerari letterari del Romanticismo inglese I Imagining Italy: Literary Itineraries in British Romanticism, a cura di L. M. Crisafulli, Bologna, Clueb, 2002, pp. 233-250; L. M. Crisafulli, "An Infernal Triangle»: Foscolo, Hobhouse, Di Breme and the Italian Context of the "Essay on the Present Literature of Italy", ivi, pp. 25I-285; A. Bruni, Foscolo, la misura del saggio, in Id., Belle vergini. "Le Grazie» tra Canova e Foscolo, Bologna, il Mulino, 2009, pp. II5-I36.

24. Italian Tragedy, I820, cit., p. IO2.

25. Si vedano la lettera $n^{\circ} 2668$ di Foscolo al Milman del 29 maggio i822 e la lettera $n^{\circ} 2700$ del Milman a Foscolo del I3 settembre I822, che si leggono in Ep. IX, rispettivamente alle pp. 62-63 e IO2. È probabilmente di questo periodo anche la lettera $\mathrm{n}^{\circ} 2353$ a Isabella Teotochi Albrizzi, pubblicata in Ep. VII, pp. 476-477, con la data congetturale I8I8; nel presentare alla Albrizzi il Milman, infatti, Foscolo lo dice, oltre che «celebre poeta di tragedie», anche "professore di Poesia nell'Università di Oxford», cattedra che lo scrittore inglese aveva ottenuto nel I82I.

26. Nella lettera $\mathrm{n}^{\circ} \mathbf{2 8 8 4}$ databile al $\mathrm{I} 823$, Milman nota due errori di stampa nella sua traduzione: «Page 97 in the last word of my translation round should be $w$ ound. I think also that there should be a comma at the end of the third line, but it is not very material» (Ep. IX, pp. 33I-332). La collaborazione tra Foscolo e Milman dovette iniziare almeno nella prima metà del I822, visto che nella lettera del 29 maggio I822, citata sopra, Foscolo parla della nuova edizione degli Essays come di un lavoro ancora da terminare, ma in buono stato di avanzamento, 
dell'articolo della «Quarterly Review», in ogni caso, rivela che Milman doveva essere in rapporti con Foscolo fin dal I820. Anzitutto, egli menziona e discute l'Ajace: pur apprezzandone alcuni versi (pronunciati dal personaggio di Tecmessa), che sarebbero «more in the true character of Greek dramatic poetry, than any perhaps with which we are acquainted in modem language», Milman critica sia la scelta del soggetto classico, che nulla ha da dire al lettore incolto e che costringe quello cólto a un confronto continuo con i precedenti, sia il fondamentale impianto allegorico (il personaggio di Agamennone adombrerebbe la figura di Napoleone, mentre il personaggio di Aiace adombrerebbe quella del generale Moreau), che impone allo spettatore «a new distraction» chiamandolo a interrogarsi sul possibile significato politico di ogni passaggio dell'opera, piuttosto che sulla sua intrinseca bellezza. Rappresentato alla Scala di Milano nel dicembre I8II e sùbito vietato dalla censura ${ }^{27}$, l'Ajace non era ancora stato dato alle stampe ${ }^{28}$, sicché, al di là delle notizie che Milman poteva leggere nell'Essay on the Present Literature of Italy ${ }^{29}$, è probabile che lo scrittore inglese avesse letto l'inedita tragedia sul manoscritto consegnatogli da Foscolo stesso ${ }^{30}$. Inoltre, come ha rilevato Stefano Carrai, un indizio ulteriore del fatto che i due scrittori si conoscessero all'altezza del I820, e che il Milman avesse avuto la possibilità di concertare con lui l'operazione dell'articolo Italian Tragedy, si evince dal fatto che concludendo la parte dedicata al Carmagnola, si augurava che «in avvenire Manzoni facesse dono ai suoi lettori di "splendide odi" anziché disgustarli con "difettose tragedie"». Ora, conclude Carrai, "poiché nessuna ode di Manzoni era allora a stampa né ancora esistevano le due celebri che egli avrebbe scritto nella primavera successiva, [...] è ragionevole attribuire ad un suo [cioè del Foscolo] suggerimento anche questo indiretto invito a

tanto da essere prossimo alla pubblicazione e, dunque, all'invio al Milman stesso: «Dear Milman—Hearing from your friend M. ${ }^{r}$ Harness that you set off on Sunday next, I hasten to send the letters which I delayed in the hope of adding to them the Essays on Petrarch. The edition, however, goes on more slowly than I thought, and you shall have them on your return" (ibid., p. 62).

27. Sulla vicenda si veda A. Manzi, Foscolo e la censura teatrale e il governo italico (con documenti d'Archivio inediti e rari), "Rivista d'Italia», XV, I8I2, $\mathrm{n}^{\circ} \mathrm{I}$, pp. 565-656, e $\mathrm{n}^{\mathrm{i}}$ 4-5, pp. 764-822.

28. Sulle vicende editoriali dell'Ajace, che non fu mai pubblicato in vita da Foscolo, si veda, oltre all'introduzione di Bèzzola a EN II, pp. XviII-XxxvıII, e alla Scheda introduttiva di M. M. Lombardi in U. Foscolo, Opere, cit., vol. I, pp. 788-796, il classico studio di G. A. Martinetti, Sul testo delle tragedie di U. Foscolo, "Giornale storico della letteratura italiana», XII, I894, n $\mathrm{n}^{\mathrm{i}}$ 67-68, pp. 7I-83, in particolare p. 75, pp. 208-23I, in particolare pp. 220-225.

29. U. Foscolo, Opere, Milano-Napoli, cit., pp. I552-I553. L'articolo di Milman cita un passaggio dell'Essay relativo ad Alfieri e Monti: «While Alfieri, in the words of Mr. Hobhouse, "was regarded as a wild irregular genius, scarcely within the pale of literary civilization, Monti was the tragic writer of Italy, and was confidently hailed as the successful candidate for an eminence as yet never occupied" (Italian Tragedy, I820, cit., p. 83).

30. Come già osservò Gambarin, Ancora del Foscolo, cit., p. 78. 
scrivere odi anziché tragedie» ${ }^{3}$. Se, infatti, l'espressione "odes» non si riferisce impropriamente agli Inni sacri, i componimenti cui l'autore dell'articolo allude non possono essere altro che le ben più 'foscoliane' odi giovanili di Manzoni, che Foscolo aveva visto nascere nei primi anni milanesi e che il più giovane poeta doveva avere in parte distrutto.

Insomma, l'ipotesi che Italian Tragedy sia il frutto di una collaborazione tra Milman e Foscolo, sul modello dell'operazione a quattro mani compiuta con Hobhouse per l'Essay on the Present Literature of Italy del 'ı8, pare degna di considerazione. La questione è, però, più complessa. Anzitutto perché, se la stroncatura del Carmagnola rispecchia l'opinione che Foscolo avrebbe espresso sulla tragedia manzoniana nelle pagine poi pubblicate dall'Orlandini con il titolo Della nuova scuola drammatica, l'entusiastico parere dato dall'estensore dell'articolo sulla Francesca da Rimini non sembra accordarsi con il giudizio poco indulgente reso a suo tempo da Foscolo al Pellico sulla sua tragedia, che egli lesse e annotò nella sua prima redazione ${ }^{22}$. Sicché, se non altro per ragioni di prudenza, la parte di Foscolo nella stesura di Italian Tragedy si configurerebbe, almeno ai fini della tesi sostenuta nell'articolo, come meno preponderante che in quella dell'Essay. Inoltre, la questione della paternità degli squarci di traduzione della Francesca da Rimini, a suo tempo attribuiti a Lord Byron e, come mostrato da Beatrice Corrigan, probabilmente ascrivibili a Hobhouse o, tutt'al più, a una collaborazione tra questi e Lord Byron (che avevano iniziato a tradurre la tragedia insieme a Milano nel I8I6) ${ }^{33}$, induce ad allargare un poco lo sguardo anche al di là della coppia Milman-Foscolo. Proprio Hobhouse, al principio del I820, aveva infatti chiesto a Foscolo di fornirgli materiali sulla tragedia, che a quanto pare Foscolo aveva promesso di trasmettergli:

Souvenez vous de la promesse que vous m'avez faite de me donner une page ou deux d'aperçus sur la tragédie. Cela me seroit de la dernière utilité à l'heure qu'il est — et il ne serait que l'affaire d'une heure pour un homme tel que vous - pensez y je vous en prie. ${ }^{34}$

L'interesse di Hobhouse per il tema, e la richiesta di assistenza al Foscolo, risalivano però all'anno precedente; in una lettera del 24 giugno I8I9 il poeta prometteva all'amico di fornirgli il suo aiuto, una volta che questi

3I. S. Carrai, Foscolo milanese tra Manzoni e Pellico, "Giornale storico della letteratura italiana», CLXXIV, I997, $\mathrm{n}^{\circ} 567$, pp. 32I-348, in particolare p. 336.

32. Ivi, pp. 343-345, con rimando a D. Chiattone, I due codici manoscritti della «Francesca da Rimini» di Silvio Pellico, «Piccolo archivio storico dell'antico marchesato di Saluzzo», I, I9OI, pp. 7I-I22, e EN VIII, p. 404.

33. B. Corrigan, Pellico's Francesca da Rimini, cit., pp. 2I6-2I8.

34. Cfr. lett. $\mathrm{n}^{\circ} 2455, E p$. VIII, p. I2I. 
gli avesse comunicato le idee di fondo della «dissertation à l'égard du théâtre Italien» che stava progettando, a probabile corollario della traduzione inglese della Francesca da Rimini di Pellico ${ }^{35}$.

Dopo il comune soggiorno milanese, Byron abbandonò presto il lavoro di traduzione della Francesca iniziato a quattro mani con Hobhouse. Esso fu probabilmente limitato ai giorni che seguirono la conoscenza di Pellico a casa del Di Breme, il 17 ottobre I8I6. Nel suo diario, in data 22 ottobre, Hobhouse annota che «Byron and I translated part of Francesca da Rimini» ${ }^{36}$; mentre una nota inedita del 30 ottobre recita "Raining - finished Francesca da Rimini», con riferimento, come si è supposto ${ }^{37}$, alla versione completa del solo primo atto oppure al completamento di un primo getto della traduzione dell'intera tragedia. A favore di questa seconda ipotesi si può allegare la testimonianza di Pietro Maroncelli: nell'introduzione alle sue Annotazioni racconta che, ottenuto da Pellico il manoscritto dell'ancora inedita Francesca da Rimini (poi pubblicata dal Di Breme insieme alla traduzione in prosa che Pellico aveva fatto del Manfred di Byron) ${ }^{38}$, lo scrittore inglese lo restituì all'autore dopo due soli giorni affermando di aver tradotto l'opera in versi ${ }^{39}$. Come che sia, in seguito Hobhouse proseguì da solo nell'impresa, con l'idea di pubblicare la traduzione - accordi in tal senso furono presi sempre con il Murray, probabilmente tra il $\mathrm{I} 820$ e il $\mathrm{I} 82 \mathrm{I}^{40}$ — e di farla precedere da un' introduzione sul teatro tragico italiano, per la quale aveva chiesto l'aiuto sia di Foscolo, come abbiamo visto, che di Lord Byron, secondo quanto testimoniano due lettere all'amico del $\mathrm{I} 2$ agosto e del 24 settembre $182 \mathrm{I}^{4 \mathrm{I}}$. Tale introduzione era forse stata concepita con un 'taglio' comparatistico, alla luce anche della recente o imminente pubblicazione, sempre per i tipi di Murray, delle tragedie di Byron Marino Faliero, Sardanapalus, The Two Foscari e Cain, che

35. Cfr. la lett. $\mathrm{n}^{\circ} 2406$, ivi, pp. 62-63.

36. Lord Broughton [J. C. Hobhouse], Recollections of a Long Life, vol. II, London, J. Murray, I909, p. 52.

37. B. Corrigan, The Byron - Hobhouse Translations of Pellico's "Francesca», «Italica», XXXV, 1958, n 4, pp. 23524I, in particolare pp. 239-240.

38. G. G. Byron, Manfredo. Poema drammatico di... Versione in prosa di Silvio Pellico, in S. Pellico, Francesca da Rimini. Tragedia di..., Milano, co' tipi di Giovanni Pirotta, I8I8.

39. «Silvio aveva tradotto il Manfred di Byron. - Byron, dimandato il manoscritto della Francesca (che solamente si recitava, e non era ancora stampata), la ebbe, e di là a due giorni, restituendolo, disse: "Non vi spiaccia se l'ho tradotta". — Tradusse in versi: "Voi pure avreste dovuto tradurre il Manfred in versi". Ma Silvio si oppose, credendo che (almeno in lingua come la nostra) non si possa far ciò senza tanto aggiungere e tanto levare all'autore originale, da non restare più quello. Ludovico Breme fece poi nel I8ıg una edizione, in cui unì la Francesca di Silvio e la suaccennata traduzione del Manfred di Byron" (Opere complete di Silvio Pellico con le addizioni alle Mie Prigioni di Piero Maroncelli, Milano, Francesco Pagnoni, I873, p. XVII).

40. Cfr. la lettera senza data di Murray a Hobhouse, provvista della sola indicazione "Friday", in B. Corrigan, The Byron-Hobhouse Translations, cit., p. 236.

4I. Ivi, p. 238. 
l'autore riconosceva fondate più sul modello della scuola italiana di Alfieri, seguìta anche da Foscolo e Pellico, che di quella inglese di Shakespeare e Johnson, per tornare agli autori citati da Milman in Italian Tragedy. Proprio Byron, infatti, nel rinunciare a difendere il proprio 'sistema drammatico' (fondato "upon the Alfieri school») dalle critiche che gli erano state mosse, scriveva al Murray, il 20 settembre I82I, che tale compito sarebbe stato svolto meglio da Hobhouse nella prefazione a "Rimini» (un titolo che potrebbe indicare sia la Francesca da Rimini del Pellico, sia una tragedia sullo stesso soggetto che, al principio del I82I, Lord Byron aveva progettato di comporre e che invece, come il Tiberius, non scrisse mai) ${ }^{42}$.

Purtroppo, tra le carte di Hobhouse la traduzione della Francesca da Rimini non è stata ritrovata. Nel I820, però, essa era quasi completata, come testimonia una lettera senza data di Murray a Hobhouse, con ogni probabilità precedente l'autunno di quello stesso anno. Nel domandare a Hobhouse di prestargli la sua copia della tragedia del Pellico, Murray lo pregava di trasmettergli anche la sua versione inglese dell'opera, che avrebbe potuto essere molto utile nella stesura di un articolo "on Foscolo» che era in fase di preparazione alla «Quarterly Review». È degno di nota che Murray, il quale scrive a Hobhouse usando la prima persona singolare $I$, utilizzi in questo passaggio la prima persona plurale $W e$, che sembra fare riferimento a una sorta di équipe di lavoro, la quale, oltre che a discorrere dell'opera di Foscolo, nello scritto avrebbe voluto dare «some notice» anche della Francesca di Pellico, con l'aggiunta di qualche squarcio della traduzione inglese che Murray chiedeva a Hobhouse di poter citare ${ }^{43}$. Il progetto cui Murray fa riferimento è da identificare ragionevolmente nella prima fase dell'operazione che avrebbe portato alla pubblicazione dell'articolo Italian Tragedy, steso poi dal Milman; un progetto nato, dunque, come un articolo su Foscolo - che proprio per i tipi di Murray, come si è ricordato, aveva appena pubblicato, o era in procinto di pubblicare, la Ricciarda - e dietro al quale Hobhouse intravedeva la partecipazione di Foscolo stesso («You are exceedingly welcome to use of the original and of the translation too if you or Mr Foscolo can turn it to any account») ${ }^{44}$. Nella risposta al Murray, Hobhouse non solo prometteva di inviargli al

42. Cfr. E. R. Vincent, Byron, Hobhouse and Foscolo. New Documents in the History of a Collaboration, Cambridge, Cambridge University Press, 1949, pp. I05-I06, e B. Corrigan, Pellico's "Francesca da Rimini», cit., p. 22I. Sulla mai scritta Francesca da Rimini di Byron si veda P. Quennell, Byron: a Self-Portrait, vol. II, London, J. Murray, 1950, p. 576.

43. Si tratta di una lettera senza data, con la sola indicazione del giorno della settimana: "Thursday». La si legge in B. Corrigan, The Byron-Hobhouse Translations, cit., pp. 235-236.

44. La risposta, senza data, dell'Hobhouse, si legge nella stessa p. 236. 
più presto sia la tragedia di Pellico sia la sua traduzione, che gli concedeva di utilizzare, ma parlava anche del progetto di stendere «a short account of Silvio Pellico» e, soprattutto, di tradurre la Ricciarda, inducendo a congetturare, diversamente da quanto pensava Mary Graham, che siano sue anche le traduzioni dei brani della tragedia foscoliana incluse in Italian Traged ${ }^{45}$. Non vi sono prove che Hobhouse abbia poi davvero consegnato al Murray la sua traduzione; tuttavia, come ha illustrato la Corrigan, allo stato attuale delle nostre conoscenze considerare gli squarci di versione della Francesca come opera di Hobhouse rappresenta l'opzione più verosimile ed economica. Che questi abbia accettato di collaborare con Murray e Milman, infatti, non stupisce: tutti e tre facevano parte della stessa cerchia di letterati e intellettuali, legati dai medesimi interessi e da una consuetudine di rapporti.

Quanto a Foscolo, che Hobhouse mostrava di considerare parte attiva nel progetto del Murray di un articolo su di lui, il suo ruolo di 'consulente' va dato ormai per assodato: è certo che esso dovette concretarsi in una serie di suggerimenti e nella partecipazione al comune dibattito sul tema del saggio, oltre che nella segnalazione e nella trasmissione di materiali, tra cui spiccano, a conferma della sua collaborazione, i riferimenti a testi inediti come l'Ajace e le odi giovanili di Manzoni. Allo stesso modo in cui richiese a Hobhouse la tragedia di Pellico e il manoscritto della sua versione, infatti, è probabile che Murray richiedesse a Foscolo il manoscritto dell'Ajace. Né si può dubitare del fatto che, predisponendosi a pubblicare la Ricciarda, Murray e i suoi sodali si intrattenessero a discutere col Foscolo del teatro tragico, tanto più che dall'Italia arrivavano notizie sia della pubblicazione del Carmagnola manzoniano, tragedia italiana vicina alle "tramontane notions of dramatic liberty» ${ }^{46}$, sia di un forte interesse di Lord Byron - alle prese, nella prima parte dell'estate, con il Marino Faliero - per il genere drammatico, secondo un gusto classicista che guardava (salvo che per la scelta dei soggetti) alla «Alfieri School» ${ }^{47}$.

Come abbiamo già osservato tuttavia, alcune opinioni contenute nell'articolo, in parte discordanti da quelle di Foscolo, inducono a escludere una sua più ampia collaborazione: la critica mossa all'Ajace, lo stesso invito a lui rivolto a scrivere un'opera che s'innalzi al di sopra dell'Ortis e della Ricciarda, l'elogio della Francesca da Rimini di Pellico, cui sembrerebbe attribuita la palma della migliore tragedia italiana contemporanea, suggeriscono

46. Italian Tragedy, I820, cit., p. 87.

47. E. R. Vincent, Byron, Hobhouse and Foscolo, cit., p. Io6. 
infatti di escludere che, come avvenuto per l'Essay on the Present Literature of Italy, egli agisse quale 'autore-ombra' del contributo. È molto probabile che le polemiche e le tensioni innescate dall'Essay nel i8ı8 lo abbiano persuaso a non riproporre un'operazione simile, difficile ormai da mascherare ai lettori italiani; inoltre, l'avere a suo tempo bollato la disputa tra classici e romantici, sempre nell' Essay, come «an idle inquiry» fu forse un motivo sufficiente per non entrare direttamente nella polemica.

Si può dunque affermare che l'articolo Italian Tragedy, uscito sulla "Quarterly Review» nell'autunno del I820, nacque probabilmente come progetto collettivo elaborato dalla cerchia di John Murray, editore della rivista, di Byron e di Foscolo; che della sua stesura così come, verosimilmente, della maggior parte degli squarci di traduzione dalle tragedie italiane esaminate - compresi quelli della Ricciarda foscoliana, secondo la testimonianza di Mary Graham, corrispondente inglese del poeta italiano - si incaricò il letterato e tragediografo Henry Hart Milman; ma che le versioni della Francesca da Rimini di Pellico furono, con ogni probabilità, opera di John Cam Hobhouse: fu infatti lo stesso Murray a chiedere a Hobhouse di poter vedere e citare la sua traduzione, che questi aveva iniziato insieme a Lord Byron quattro anni prima durante il comune soggiorno milanese. È possibile, anche se non si può accertare, che il contributo di Hobhouse sia andato oltre la traduzione della Francesca, estendendosi alla traduzione di alcuni passi della Ricciarda (ma su questo punto pare più prudente attenersi alla testimonianza di Mary Graham) e ad alcuni materiali sul Pellico, che egli aveva conosciuto a Milano a casa di Ludovico di Breme, insieme a Monti e a numerosi altri intellettuali italiani. In particolare, pare si possa riferire all'opinione di Hobhouse un passaggio dell'articolo Italian Tragedy relativo al Pellico, che precede di poco l'indirizzo al Foscolo su cui si chiude lo scritto. L'estensore del saggio, infatti, auspica che Pellico possa pubblicare presto una nuova opera, che di certo uno tra gli scrittori inglesi sarà felice di tradurre, così come l'autore italiano si era impegnato a tradurre il Manfred di Lord Byron, appena pubblicato dal Murray, e a farlo conoscere al pubblico italiano. La traduzione, allegata alla Francesca da Rimini nella prima edizione della tragedia (apparsa, come si è visto, a Milano per i tipi di Pirotta nel I8I8, per cura del Di Breme) ${ }^{48}$, era in prosa, mentre secondo l'autore dell'articolo meglio avrebbe fatto Pellico a renderla in versi ${ }^{49}$. Come già

48. Il Manfred era stato pubblicato dal Murray l'anno precedente: G. G. Byron, Manfred, a Dramatic Poem, London, John Murray, I8I7.

49. Italian Tragedy, I820, cit., p. IOI. 
notava la Lograsso ${ }^{5 \circ}$, l'affermazione è sorprendentemente simile a quanto Byron, secondo il racconto del Maroncelli, ebbe modo di dire a Pellico a Milano nel 1816, restituendogli il manoscritto della Francesca da Rimini che lui e Hobhouse avevano tradotto: “"Non vi spiaccia se l'ho tradotta”. Tradusse in versi: "Voi pure avreste dovuto tradurre il Manfred in versi" "sI. Se anche l'affermazione del saggio non risale direttamente a Hobhouse, essa certo rispecchia il pensiero suo e di Lord Byron, a riprova della circolazione e discussione di idee sul teatro tragico e sulla traduzione (altro tema caro a Foscolo) che dovette precedere la pubblicazione dell'articolo Italian Traged $y^{52}$. Analogo discorso vale per l'invito rivolto a Foscolo ad affidare la propria fama futura a un'opera più grande dell'Ortis o della Ricciarda, che trova perfetta rispondenza in un passaggio di una lettera di Byron a Murray del 6 aprile i8I9:

Why does he not do something more than the Letters of Ortis and a tragedy and pamphlets? He has good fifteen years more at his command than I have: what has he done all that time? - proved his genius, doubtless, but not fixed its fame, nor done his utmost ${ }^{\dagger 3}$.

Quanto a Foscolo, se l'esperienza dell'Essay lo dissuase certamente dal riproporre l'espediente dell' autore ombra', è verosimile che, anche per ragioni di difesa e accrescimento del proprio prestigio in terra inglese, si facesse nel 1820 motore del dibattito inglese sul teatro tragico, come reazione al clamore suscitato, non ultimo per lo zelo dei romantici italiani, dalla pubblicazione del Carmagnola del Manzoni e dai contenuti della sua prefazione.

Piuttosto che entrare nell'agone della disputa tra classicisti e romantici sul rispetto delle unità aristoteliche e sul rapporto tra poesia e storia, suscitato dalla prefazione manzoniana, e sulla questione della scelta dei soggetti tragici, Foscolo intervenne nel dibattito pubblicando in tutta fretta una propria tragedia, la Ricciarda (e non, si badi, il classicistico, allegorico e parimenti inedito Ajace), composta e portata in scena nel I8I3, che di per sé illustrava un preciso piano letterario, elaborato molto tempo prima tanto di

50. A. Lograsso, Byron traduttore, cit., pp. 234-235.

5I. Pellico, Opere complete, cit., p. XI.

52. Cfr. A. Lograsso, Byron traduttore, cit., p. 245: «E questa opinione coincide esattamente con quella di Byron così come è riferita dal Maroncelli nelle Addizioni: non vi è dubbio quindi che l'articolo rifletta qua e là le opinioni di Byron e quelle di Hobhouse».

53. Il passo, che si legge in Letters and Journals of Lord Byron, with Notices of his Life, by Th. Moore, 2 voll., A. and W. Galignani, Paris, I830, vol. II, p. 296, è citato da S. Parmegiani, Ugo Foscolo and English Culture, London, Legenda, 20II, p. I22. Nell'articolo Italian Tragedy, I820, cit., p. IOI, si legge: «to him we would say, that the name of Foscolo should be known to posterity as something greater than that of the author of Ortis's Letters, or even of Ricciarda». 
Manzoni quanto di Pellico. Rispettando le unità aristoteliche la Ricciarda si inseriva nella tradizione della "Alfieri School»; da un lato, infatti, la scelta del soggetto medievale italiano si giovava dei precedenti alfieriani del Don Garzia, de La congiura de’ Pazzi e, soprattutto, della Rosmunda, la prima tragedia alfieriana costruita rigorosamente con soli quattro personaggi, le cui vicende dall'evidente risvolto 'patriottico' come nella Ricciarda, pur essendo basate su antecedenti storici reali, erano frutto dell'invenzione dell'autore (e non sarà certo necessario tornare qui sull'importanza che la Rosmunda ebbe anche nell'elaborazione dell'Adelchi) ${ }^{54}$; dall'altro, anticipava l'opzione dantesca della Francesca da Rimini e il 'gusto' sia della futura generazione romantica sia, soprattutto, della cerchia byroniana, mentre l'invenzione della vicenda (vagamente shakespeariana) di Guido e Ricciarda e dei loro padri Averardo e Guelfo — che, errando, l'autore di Italian Tragedy credeva "founded on history» 55 - manifestava la netta predilezione dell'autore per le ragioni della poesia su quelle della storia.

Tuttavia, con Pellico rinchiuso allo Spielberg e Byron lontano dalla madrepatria e prossimo a trovare la morte in Grecia, la rapida eclissi di Foscolo dalla società aristocratica inglese e dalla scena letteraria europea, dovuta ai noti problemi finanziari cui andò incontro, lasciò 'campo aperto' all'affermazione anche oltremanica di Manzoni, che nel frattempo aveva portato a termine e pubblicato a Milano l'Adelchi (I822). Datosi al volontario esilio e caduto in disgrazia, privo del sostegno della nuova generazione di letterati italiani e dell'appoggio degli influenti personaggi inglesi che lo avevano accolto e promosso nei primi anni londinesi, Foscolo, che dopo la Ricciarda non aveva più pubblicato nuove opere 'd'invenzione', sembra ormai cancellato - persino da un punto di vista inglese - dalla scena letteraria italiana ed europea, di cui era stato fino a poco tempo prima un indiscusso protagonista. Non una parola fu spesa per lui né nella recensione al Manzoni apparsa sul numero di dicembre I826 della «Monthly Review» (nella quale il Pellico, «the ill-fated author of Francesca and Eufemio», ottiene almeno una veloce menzione tra $\mathrm{i}$ «favourite writers of the day», insieme a Grossi, Sestini, Bertolotti, Torti, Visconti, Pindemonte e soprattutto Monti) ${ }^{56}$, né nel già citato articolo Italian Tragedy pubblicato nel luglio del I827 sulla «Foreign Quarterly Review».

54. Per un'analisi d'insieme della Rosmunda, si veda almeno la sintesi che offre L. Melosi, Paragrafi sulla "Rosmunda», in R. Turchi (a cura di), Alfieri tragico, Firenze, Le Lettere, 2004 ("La Rassegna della Letteratura italiana", CVII, nº 2), pp. 524-540.

55. Italian Tragedy, I820, cit. p. IOI.

56. The Works of Manzoni, "The Monthly Review», n.s., III, I826, December, pp. 482-493. 
Tutto ciò spiega forse perché proprio nella seconda metà del I826, dopo avere superato il periodo più buio dell'esilio inglese, sollecitato dal Bowring e dalla pubblicazione dell'edizione Molini delle Tragedie manzoniane, Foscolo sentisse la necessità di ritornare in prima persona sul tema del genere drammatico, della poetica tragica di Manzoni e dei giudizi, mai condivisi, di Goethe, e si applicasse alla stesura dell'ambizioso saggio On Literary Criticism, che non terminò mai e che l'Orlandini «rese noto come meglio poté o seppe» ${ }^{57}$ dopo la morte del poeta con il titolo Della nuova scuola drammatica italiana. Per Foscolo, che per un paio d'anni si era nascosto agli occhi di creditori, colleghi e amici, si trattava di una questione rimasta aperta, resa ancora più attuale e bruciante dalla scomparsa del suo nome dalla critica contemporanea. Manzoni invece, approdato già da tempo al romanzo storico e in procinto di pubblicare la Ventisettana, era ormai ben al di là sia della disputa tra classici e romantici sia del dibattito sul teatro tragico, nel quale, grazie anche all'autorevole sostegno di Goethe e Fauriel, era risultato trionfatore, almeno sul piano della poetica drammatica. A quell'altezza temporale il lucido intervento di Foscolo, «severo, ma non ingiurioso», era per così dire un frutto fuori stagione; sicché nel I85I Gino Capponi, nell'esortare Orlandini a pubblicare l'inedito scritto emerso dalle carte inglesi dell'amico, poteva giudicarlo «opera d'un forte ingegno» e allo stesso tempo «roba d'un altro tempo: [...] roba che parla altra lingua, perché alla fine ogni tempo ha le sue corbellerie» ${ }^{58}$.

L'incompiuto saggio di Foscolo sul genere drammatico meriterebbe un rinnovato interesse ecdotico, che chiarisca i rapporti tra le diverse redazioni presenti tra le carte foscoliane conservate presso la Biblioteca Labronica e porti a riflettere sulla genesi, lo sviluppo e la destinazione dello scritto ${ }^{59}$. Sul testo di quell'importante contributo, e sui suoi contenuti, avremo forse modo di ritornare più nel dettaglio in un'altra sede.

57. G. Gambarin, Ancora del Foscolo, cit., p. 8o.

58. Lettera del $\mathrm{I}^{\circ}$ agosto I85I, citata dal Gambarin, ibid.

59. Sulla necessità di rimettere mano all'edizione del Foscolo dell'esilio londinese ci permettiamo di rimandare a P. Borsa, Per l'edizione del Foscolo «inglese», in P. Chiesa e A. Cadioli (a cura di), Prassi ecdotiche, Milano, Cisalpino, 2008, pp. 299-335; Id., Appunti per l'edizione delle "Epoche della lingua italiana» di Ugo Foscolo, "Studi italiani», XLVII-XLVIII, 20I3, n. I-2, pp. I23-I49; e U. Foscolo, Antiquarj e Critici. On the Antiquarians and Critics, edizione critica bilingue a cura di P. Borsa, Milano, Ledizioni, 2012. 